\title{
Observations of comets and minor planets at Kiev comets station (585)
}

\author{
Alexander R. Baransky, Klim I. Churuymov, \\ and Vasyl A. Ponomarenko
}

Astronomical Observatory, Kyiv Shevchenko National University, Box 04053, Observatorna str., 3, Kyiv, Ukraine

email: klim.churyumov@observ.univ.kiev.ua

\begin{abstract}
We present the results of astrometric and photometric observations of comets and minor planets obtained at the Kiev comet station (Code MPC 585) of the Astronomical Observatory of Kyiv Shevchenko National University in 2006-2009. The 2318 position observations of 176 comets, 302 observations of 57 numbered minor planets, and 220 observations of 30 unnumbered minor planets were obtained. The accuracy of the astrometric observations of the comets is analyzed.
\end{abstract}

Keywords. Astrometry, ephemeris, comets: general, minor planets

\section{Introduction}

CCD astrometric monitoring of new and short period comets and new asteroids is very important for the determination and improvement of their orbits and the study of the orbital evolution of new small bodies of the Solar System (Steel \& Marsden, 1996). In 2006 a programme was started at observation station of the Astronomical observatory of the Kiev National University in Lisnyky ("Kiev Comet Station, code MPC 585 performing astrometric and photometric observations of comets and minor planets of Solar System. Observations are obtained according to the technique of the Minor Planet Center (MPC) (Holmes, 1995) with of the telescope reflector AZT-8 (D= $0.7 \mathrm{~m}$ ), and CCD ST-8E which is accomodated in the primary focus of the telescope (focus of system $F=2.8 \mathrm{~m}$, focal ratio $f / 4$ ). The above noted equipment gives the chance to detect in integrated light the images of asteroids to a brightness of $21^{m}$, and comets to $19.5^{m}$.

\section{The program of monitoring}

The programme of monitoring minor Solar System bodies is directed to the objectives: 1) Observations of just discovered objects for the purpose of confirming their actually existence and receiving first astrometric and photometric observations. The list of objects which require confirmation is daily renewed by the Minor Planet Centre internet page - The NEO Confirmation Page. 2) Astrometric observations of known long- and short-period comets for the purpose of determining changes in the orbital characteristics which are connected to non-gravitational and gravitational perturbations. 3) Photometric observations of comets (integral and nucleus comet magnitude, size of the coma, degree of a central condensation of in the coma, length and position angle of the tail). 

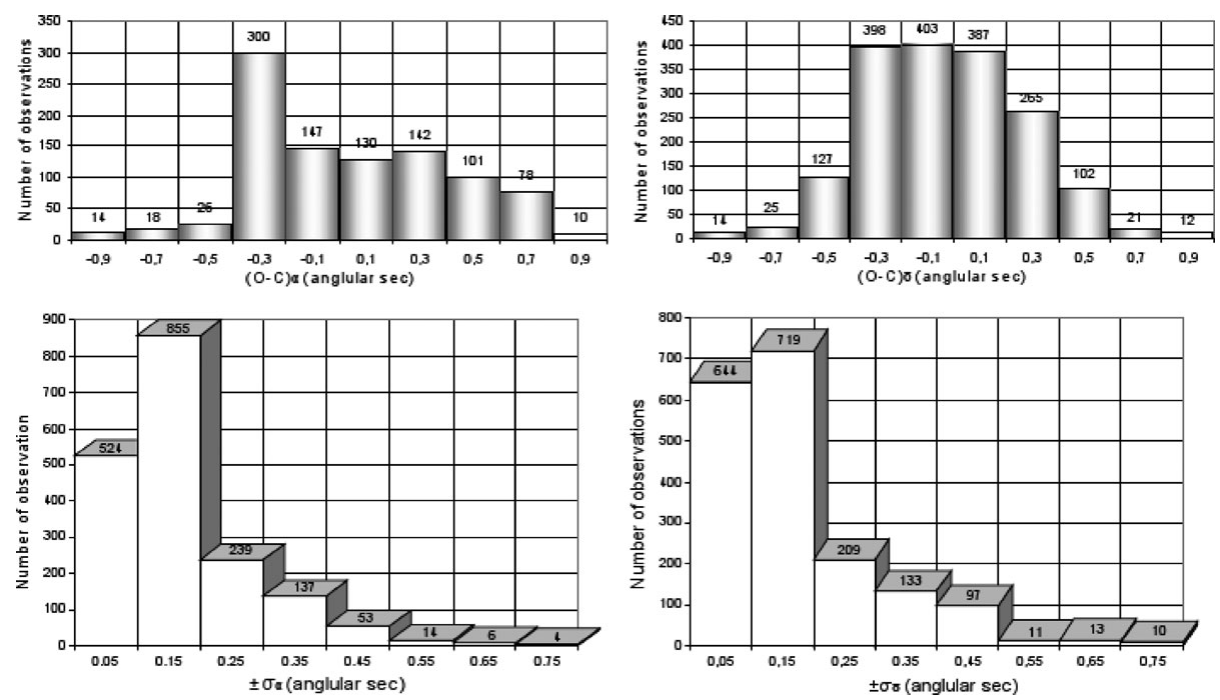

Figure 1. Distributions of the residuals $(O-C)_{\alpha} \pm \sigma_{\alpha} \&(O-C)_{\delta} \pm \alpha_{\delta}$.

4) Observations of unique comets during rare outbursts, split comets, appearance of peculiar tails. For example, we obtained a long series of observations of the unique comet $73 \mathrm{P} /$ Schwassmann-Wachmann, which nucleus had split into a considerable quantity of fragments (we identified 16 separate fragments of the comet). 5) Astrometric and photometric observations of asteroids which approach to the Earth (NEOs) and asteroids of the Main Belt.

\section{Result and analyses of observations}

The astrometric observations of comets and their accuracy are shown in the table 4

Figure (Fig. 1) shows diagrams of the accuray distribution of the observed (O-C) in angular sec for Right Accession $(O-C)_{\alpha} \pm \sigma_{\alpha}$ and for Declination $(O-C)_{\delta} \pm \sigma_{\delta}$. It is seen, that the basic quantity of observations is received with an accuracy of 0 0.4 " for $(O-C)_{\alpha}$ and $(O-C)_{\delta}$. The diagram $(O-C)_{\alpha}$ is a little asymmetric, which is probably connected with restriction of accuracy of the telescopes clock mechanism. On the diagrams for $\pm \sigma_{\alpha}$ and $\pm \sigma_{\delta}$ the error of the majority of observations does not exceed $0,2 "$.

\section{Conclusion}

Between 2.04.2006 - 9.05.2009 (140 nights) a total of 2318 observations were obtained of 176 comets, and 522 observations of 87 asteroids. The maximum quantity of observations has been obtained with an accuracy from 0,1 " to 0,2 ". Results of astrometric and photometric observations were contributed to the database of the Minor Planet Centre (MPC), the International Comet Quarterly (ICQ), and to the database of the British Astronomical Association (BAA). 
Table 1. Accuracy of astrometric observations of comets. Column 1: comet name, column 2: period of observations, column 3: number of observations, column 4: residual (O-C) for R.A., column 5: (O-C) for Declination.

\begin{tabular}{|c|c|c|c|c|}
\hline Comets & Period observ. & $\mathbf{N}$ & $(O-C)_{\alpha} \pm \sigma_{\alpha}$ & $(O-C)_{\delta} \pm \sigma_{\delta}$ \\
\hline 4P/Faye & 2006 & 30 & $0.22 \pm 0.11$ & $0.03 \pm 0.09$ \\
\hline $6 \mathrm{P} / \mathrm{d}$ Árrest & 2008 & 17 & $0.10 \pm 0.17$ & $-0.04 \pm 0.15$ \\
\hline $8 \mathrm{P} /$ Tuttle & 2008 & 8 & $-0.14 \pm 0.22$ & $0.12 \pm 0.26$ \\
\hline 17P/Holmes & $2007-2008$ & 39 & $0.31 \pm 0.11$ & $0.29 \pm 0.08$ \\
\hline 19P/Borrelly & $2008-2009$ & 4 & $-0.67 \pm 0.11$ & $0.50 \pm 0.22$ \\
\hline $22 \mathrm{P} /$ Kopff & 2009 & 18 & $-0.67 \pm 0.19$ & $-0.19 \pm 0.16$ \\
\hline 29P/Schwassmann-Wachmann & $2006,2008-2009$ & 33 & $0.15 \pm 0.16$ & $-0.07 \pm 0.18$ \\
\hline 41P/Tuttle-Giacobini-Kresak & 2006 & 2 & $0.30 \pm 1.20$ & $0.05 \pm 0.65$ \\
\hline 44P/Reinmuth & $2007-2008$ & 10 & $0.20 \pm 0.23$ & $0.25 \pm 0.20$ \\
\hline 46P/Wirtanen & $2007-2009$ & 16 & $-0.20 \pm 0.21$ & $-0.11 \pm 0.25$ \\
\hline $50 \mathrm{P} /$ Arend & $2007-2008$ & 14 & $-0.08 \pm 0.18$ & $-0.15 \pm 0.07$ \\
\hline 51P/Harrington & 2008 & 7 & $-0.23 \pm 0.28$ & $0.24 \pm 0.14$ \\
\hline 59P/Kearns-Kwee & 2008 & 10 & $-0.21 \pm 0.16$ & $-0.45 \pm 0.13$ \\
\hline $65 \mathrm{P} /$ Gunn & 2008 & 4 & $0.11 \pm 0.08$ & $-0.71 \pm 0.14$ \\
\hline 67P/Churyumov-Gerasimenko & 2009 & 9 & $0.49 \pm 0.26$ & $0.19 \pm 0.10$ \\
\hline 68P/Klemola & 2008 & 4 & $0.35 \pm 0.77$ & $-0.05 \pm 0.53$ \\
\hline 74P/Smirnova-Chernykh & $2007-2009$ & 23 & $-0.04 \pm 0.16$ & $0.19 \pm 0.09$ \\
\hline $77 \mathrm{P} /$ Longmore & 2009 & 23 & $-0.05 \pm 0.11$ & $-0.21 \pm 0.13$ \\
\hline 79P/du Toit-Hartley & 2008 & 3 & $0.17 \pm 0.18$ & $-0.77 \pm 0.37$ \\
\hline 84P/Giclas & 2006 & 9 & $-0.33 \pm 0.20$ & $-0.84 \pm 0.30$ \\
\hline 87P/Bus & 2007 & 3 & $0.77 \pm 0.35$ & $0.43 \pm 0.64$ \\
\hline 88P/Howell & 2009 & 5 & $0.52 \pm 0.07$ & $0.05 \pm 0.23$ \\
\hline 98P/Takamizawa & 2006 & 3 & $0.73 \pm 0.18$ & $0.17 \pm 0.52$ \\
\hline 110P/Hartley & $2007-2008$ & 3 & $-0.33 \pm 0.18$ & $0.30 \pm 0.36$ \\
\hline $112 \mathrm{P} /$ Urata-Niijima & 2006 & 11 & $-0.20 \pm 0.26$ & $-0.44 \pm 0.29$ \\
\hline 114P/Wiseman-Skiff & 2006 & 3 & $-0.20 \pm 0.87$ & $0.33 \pm 0.62$ \\
\hline 116P/Wild & $2008-2009$ & 8 & $0.15 \pm 0.19$ & $0.10 \pm 0.18$ \\
\hline $124 \mathrm{P} /$ Mrkos & 2008 & 10 & $-0.480 \pm 0.06$ & $0.02 \pm 0.14$ \\
\hline 128P/Shoemaker-Holt & $2006-2008$ & 2 & $0.10 \pm 0.50$ & $0.15 \pm 0.25$ \\
\hline 143P/Kowal-Mrkos & 2009 & 10 & $-0.18 \pm 0.09$ & $-0.03 \pm 0.15$ \\
\hline 144P/Kushida & $2008-2009$ & 13 & $0.64 \pm 0.29$ & $0.02 \pm 0.16$ \\
\hline 173P/Mueller & 2008 & 5 & $0.75 \pm 0.35$ & $-0.10 \pm 0.19$ \\
\hline 177P/Barnard & 2006 & 50 & $-0.32 \pm 0.06$ & $-0.41 \pm 0.10$ \\
\hline 179P/Jedicke & $2007-2008$ & 11 & $0.27 \pm 0.44$ & $-0.28 \pm 0.18$ \\
\hline 180P/NEAT & 2008 & 10 & $-0.22 \pm 0.23$ & $0.08 \pm 0.26$ \\
\hline 182P/LONEOS & 2007 & 4 & $-0.07 \pm 0.44$ & $0.15 \pm 0.72$ \\
\hline 183P/Korlevic-Juric & 2008 & 7 & $-0.65 \pm 0.13$ & $-0.62 \pm 0.03$ \\
\hline 188P/LINEAR-Mueller & 2008 & 7 & $-0.30 \pm 0.48$ & $0.10 \pm 0.23$ \\
\hline $189 \mathrm{P} / \mathrm{NEAT}=\mathrm{P} / 2007 \mathrm{~N} 2$ & 2007 & 3 & $0.00 \pm 0.40$ & $-0.17 \pm 0.87$ \\
\hline $191 \mathrm{P} /$ McNaught $=\mathrm{P} / 2007 \mathrm{~N} 1$ & 2007 & 11 & $-0.21 \pm 0.42$ & $-0.47 \pm 0.21$ \\
\hline 192P/Shoemaker-Levy & 2008 & 5 & $0.20 \pm 0.31$ & $-0.79 \pm 0.26$ \\
\hline 194P/LINEAR & 2008 & 3 & $0.18 \pm 0.05$ & $0.07 \pm 0.20$ \\
\hline 197P/LINEAR & 2008 & 6 & $0.36 \pm 0.40$ & $0.14 \pm 0.41$ \\
\hline 200P/Larsen & 2008 & 5 & $-0.03 \pm 0.39$ & $0.25 \pm 0.14$ \\
\hline $202 \mathrm{P} / \mathrm{Scotti}$ & 2008 & 5 & $-0.25 \pm 0.54$ & $-0.33 \pm 0.08$ \\
\hline 205P/Giacobini & 2008 & 28 & $-0.94 \pm 0.13$ & $-0.34 \pm 0.07$ \\
\hline 206P $/$ Barnard-Boattini $=\mathrm{P} / 2008 \mathrm{~T} 3$ & 2008 & 16 & $-0.31 \pm 0.17$ & $0.01 \pm 0.14$ \\
\hline 210P/Christensen & 2009 & 10 & $-0.23 \pm 0.11$ & $-0.27 \pm 0.07$ \\
\hline C/2002 VQ94 (LINEAR) & 2006,2008 & 4 & $0.22 \pm 0.36$ & $-0.16 \pm 0.35$ \\
\hline C/2003 WT42 (LINEAR) & $2006-2007$ & 13 & $-0.19 \pm 0.17$ & $0.10 \pm 0.17$ \\
\hline C/2004 B1 (LINEAR) & 2006 & 45 & $-0.01 \pm 0.09$ & $0.44 \pm 0.49$ \\
\hline P/2004 VR 8 (LONEOS) & 2006 & 3 & $0.18 \pm 0.22$ & $1.02 \pm 0.13$ \\
\hline P/2005 JY 126 (Catalina) & 2006 & 4 & $0.53 \pm 0.10$ & $0.15 \pm 0.23$ \\
\hline C/2005 L3 (McNaught) & $2007-2008$ & 60 & $0.38 \pm 0.07$ & $0.40 \pm 0.09$ \\
\hline C/2005 S4 (McNaught) & $2006-2008$ & 10 & $-0.09 \pm 0.17$ & $0.47 \pm 0.36$ \\
\hline
\end{tabular}




\begin{tabular}{|c|c|c|c|c|}
\hline Comets & Period observ. & $\mathbf{N}$ & $(O-C)_{\alpha} \pm \sigma_{\alpha}$ & $(O-C)_{\delta} \pm \sigma_{\delta}$ \\
\hline $\mathrm{P} / 2005$ SB 216 (LONEOS) & 2006 & 5 & $-0.24 \pm 0.20$ & $0.29 \pm 0.14$ \\
\hline C/2006 CK10 (Catalina) & 2006 & 5 & $0.46 \pm 0.20$ & $-0.4 \pm 0.80$ \\
\hline $\mathrm{P} / 2006 \mathrm{~F} 1$ (Kowalski) & 2006 & 7 & $0.88 \pm 0.26$ & $-0.00 \pm 0.31$ \\
\hline P/2006 H1 (McNaught) & 2006 & 8 & $0.64 \pm 0.30$ & $0.73 \pm 0.17$ \\
\hline P/2006 HR 30 (Siding Spring) & $2006-2007$ & 21 & $0.06 \pm 0.19$ & $-0.14 \pm 0.10$ \\
\hline $\mathrm{P} / 2006 \mathrm{~K} 2$ (McNaught) & 2006 & 3 & $0.89 \pm 0.35$ & $0.06 \pm 0.90$ \\
\hline $\mathrm{C} / 2006 \mathrm{~K} 4(\mathrm{NEAT})$ & 2006 & 4 & $0.43 \pm 0.35$ & $-0.44 \pm 0.44$ \\
\hline C/2006 M1 (LINEÁR) & 2006 & 9 & $0.02 \pm 0.14$ & $-0.07 \pm 0.21$ \\
\hline $\mathrm{C} / 2006 \mathrm{M} 4$ (SWAN) & 2006 & 4 & $-0.43 \pm 0.27$ & $-0.35 \pm 0.11$ \\
\hline C/2006 O2 (Garradd) & 2006 & 5 & $-0.20 \pm 0.31$ & $-0.23 \pm 0.50$ \\
\hline P/2006 R2 (Christensen) & 2006 & 6 & $0.10 \pm 0.41$ & $-0.64 \pm 0.37$ \\
\hline C/2006 OF2 (Broughton) & $2007-2009$ & 72 & $-0.32 \pm 0.05$ & $-0.22 \pm 0.04$ \\
\hline C/2006 Q1 (McNaught) & 2009 & 11 & $-0.68 \pm 0.24$ & $0.53 \pm 0.06$ \\
\hline P/2006 S1 (Christensen) & 2006 & 13 & $-0.18 \pm 0.21$ & $0.48 \pm 0.39$ \\
\hline C/2006 S2 (LINEAR) & 2006 & 8 & $-0.55 \pm 0.15$ & $0.69 \pm 0.24$ \\
\hline C/2006 S3 (LONEOS) & 2006 & 3 & $0.88 \pm 0.90$ & $0.02 \pm 0.28$ \\
\hline P/2006 S4 (Christensen) & 2006 & 14 & $0.38 \pm 0.27$ & $-0.10 \pm 0.33$ \\
\hline C/2006 S5 (Hill) & 2006,2008 & 34 & $0.39 \pm 0.13$ & $-0.10 \pm 0.08$ \\
\hline $\mathrm{P} / 2006$ S6 (Hill) & 2006 & 19 & $0.28 \pm 0.12$ & $0.29 \pm 0.11$ \\
\hline C/2006 V1 (Catalina) & 2007 & 4 & $0.33 \pm 0.34$ & $0.22 \pm 0.24$ \\
\hline C/2006 VZ13 (LINEAR) & 2007 & 8 & $0.54 \pm 0.30$ & $0.09 \pm 0.36$ \\
\hline C/2006 W3 (Christensen) & $2008-2009$ & 83 & $0.62 \pm 0.04$ & $1.38 \pm 0.05$ \\
\hline P/2006 U1 (LINEAR) & 2006 & 9 & $0.60 \pm 0.25$ & $-0.14 \pm 0.12$ \\
\hline P/2006 U5 (Christensen) & 2006 & 3 & $-0.17 \pm 0.05$ & $0.05 \pm 0.14$ \\
\hline C/2007 B2 (Skiff) & $2007-2008$ & 29 & $-0.24 \pm 0.09$ & $-0.20 \pm 0.09$ \\
\hline C'/2007 D1 (LINEAR) & 2007 & 7 & $0.22 \pm 0.10$ & $0.02 \pm 0.15$ \\
\hline C/2007 E1 (Garradd) & 2007 & 35 & $0.48 \pm 0.13$ & $-0.14 \pm 0.13$ \\
\hline C/2007 E2 (Lovejoy) & 2007 & 64 & $0.46 \pm 0.14$ & $-0.28 \pm 0.14$ \\
\hline C/2007 F1 (LONEOS) & 2007 & 6 & $0.05 \pm 0.32$ & $-0.27 \pm 0.36$ \\
\hline C/2007 G1 (LINEAR) & $2007-2008$ & 15 & $0.15 \pm 0.18$ & $0.12 \pm 0.10$ \\
\hline P/2007 H1 (McNaught) & 2007 & 10 & $0.15 \pm 0.17$ & $0.06 \pm 0.19$ \\
\hline C'/2007 M1 (McNaught) & 2007 & 10 & $-0.51 \pm 0.29$ & $-0.21 \pm 0.34$ \\
\hline C/2007 JA21 & 2007 & 4 & $0.12 \pm 0.54$ & $0.14 \pm 0.59$ \\
\hline C/2007 M3 (LINEAR) & 2007 & 27 & $-0.40 \pm 0.17$ & $0.02 \pm 0.07$ \\
\hline C/2007 N3 (Lulin) & $2007-2009$ & 44 & $0.01 \pm 0.11$ & $0.32 \pm 0.07$ \\
\hline C/2007 O1 (LINEAR) & 2007 & 5 & $-0.05 \pm 0.18$ & $-0.26 \pm 0.42$ \\
\hline $\mathrm{P} / 2007$ Q2 (Gilmore) & 2007 & 9 & $-0.41 \pm 0.34$ & $-0.31 \pm 0.32$ \\
\hline P/2007 R1 (Larson) & 2007 & 8 & $-0.02 \pm 0.34$ & $-0.05 \pm 0.44$ \\
\hline P/2007 R2 (Gibbs) & 2007 & 10 & $0.34 \pm 0.22$ & $-0.22 \pm 0.29$ \\
\hline P/2007 S1 (Zhao) & 2007 & 5 & $1.37 \pm 0.33$ & $-1.15 \pm 0.49$ \\
\hline C/2007 S2 (Lemmon) & 2007 & 9 & $0.33 \pm 0.17$ & $-0.19 \pm 0.39$ \\
\hline C/2007 T1 (McNaught) & 2007 & 5 & $-0.50 \pm 0.20$ & $0.62 \pm 0.23$ \\
\hline $\mathrm{P} / 2007$ T2 (Kowalski) & 2007 & 11 & $0.60 \pm 0.22$ & $0.59 \pm 0.21$ \\
\hline C/2007 T5 (Gibbs) & $2007-2008$ & 19 & $-0.28 \pm 0.37$ & $-0.41 \pm 0.45$ \\
\hline P/2007 T6 (Catalina) & 2007 & 9 & $-0.34 \pm 0.32$ & $-0.42 \pm 0.19$ \\
\hline C/2007 U1 (LINEAR) & 2008 & 10 & $0.12 \pm 0.16$ & $0.30 \pm 0.14$ \\
\hline $\mathrm{P} / 2007 \mathrm{~V} 1$ (Larson) & $2007-2008$ & 9 & $0.37 \pm 0.32$ & $0.31 \pm 0.33$ \\
\hline C/2007 W1 (Boattini) & $2007-2008$ & 30 & $-0.30 \pm 0.13$ & $-0.37 \pm 0.15$ \\
\hline C/2007 W3 (LINEAR) & 2008 & 15 & $0.65 \pm 0.32$ & $-0.23 \pm 0.36$ \\
\hline C/2007 Y1 (LINEAR) & 2008 & 28 & $-0.28 \pm 0.18$ & $0.29 \pm 0.19$ \\
\hline $\mathrm{P} / 2008$ A2 (LINEAR) & 2008 & 18 & $-0.30 \pm 0.23$ & $-0.18 \pm 0.22$ \\
\hline C/2008 C1 (Chen-Gao) & 2008 & 46 & $-0.23 \pm 0.12$ & $-0.31 \pm 0.12$ \\
\hline C/2008 E1 (Catalina) & 2008 & 6 & $0.94 \pm 0.15$ & $-0.28 \pm 0.27$ \\
\hline C/2008 FK75 (Lemmon-Siding Spring) & 2009 & 5 & $0.04 \pm 0.09$ & $1.01 \pm .096$ \\
\hline $\mathrm{C} / 2008 \mathrm{G} 1$ (Gibbs) & 2008 & 5 & $0.12 \pm 0.09$ & $0.56 \pm 0.22$ \\
\hline C'/2008 H1 (LINEÁR) & 2008 & 16 & $-0.15 \pm 0.24$ & $0.40 \pm 0.21$ \\
\hline C/2008 J1 (Boattini) & 2008 & 33 & $-0.14 \pm 0.07$ & $-0.00 \pm 0.13$ \\
\hline $\mathrm{P} / 2008$ J2 (Beshore) & 2008 & 27 & $+0.57 \pm 0.10$ & $+0.19 \pm 0.13$ \\
\hline
\end{tabular}


A. R. Baransky, K. I. Churyumov, \& V. A. Ponomarenko

\begin{tabular}{lcccc}
\hline Comets & Period observ. & $\mathbf{N}$ & $(O-C)_{\alpha} \pm \sigma_{\alpha}$ & $(O-C)_{\delta} \pm \sigma_{\delta}$ \\
\hline C/2008 J5 (Garradd) & 2008 & 5 & $-0.48 \pm 0.51$ & $0.20 \pm 0.28$ \\
C/2008 J6 (Hill) & 2008 & 22 & $0.30 \pm 0.17$ & $-0.18 \pm 0.20$ \\
P/2008 L2 (Hill) & 2008 & 13 & $0.12 \pm 0.07$ & $-0.02 \pm 0.07$ \\
C/2008 L3 (Hill) & 2008 & 4 & $1.31 \pm 0.06$ & $0.83 \pm 0.20$ \\
C/2008 N1 (Holmes) & 2008 & 9 & $-0.10 \pm 0.14$ & $0.18 \pm 0.12$ \\
P/2008 O2 (McNaught) & 2008 & 20 & $0.411 \pm 0.17$ & $0.11 \pm 0.17$ \\
C/2008 Q1 (Maticic) & $2008-2009$ & 37 & $-0.29 \pm 0.10$ & $0.11 \pm 0.11$ \\
P/2008 Q2 (Ory) & 2008 & 15 & $0.41 \pm 0.07$ & $-0.03 \pm 0.04$ \\
P/2008 QP20 (LINEAR-Hill) & 2008 & 8 & $-0.37 \pm 0.15$ & $-0.57 \pm 0.17$ \\
C/2008 R3 (LINEAR) & 2008 & 6 & $0.41 \pm 0.18$ & $-0.19 \pm 0.14$ \\
\hline
\end{tabular}

\section{References}

Steel, D. I. \& Marsden, B. G. 1996, Earth, Moon, and Planets, 74, 2

Holmes, A. 1995, CCD Astronomy, 2, 1 